\title{
ELECTIONS TO MEMBERSHIP
}

\section{Ordinary Membership}

Ahmad, Kathleen Kemp, Pa St U, University Park, PA

Allen, A D, Algorithms, Northridge, CA

Amayo, Ralph, U of Conn, Storrs, CT

Andry, Albert N, Mech Eng Dept, East Lansing, MI

Bart, H, Vrye U, Amsterdam, Netherlands

Beckner, William, U of Chicago, Chicago, IL

Behrens, Kenneth, 618 Gifford, Syracuse, NY

Ben Lashiher, Ali M, Arab Development Inst, Tripoli, Libya

Bennison, Victor Lawrence, $U$ of Chicago, Chicago, IL

Berg, H L, 4337 Bagley Parkway, Madison, WI

Bertman, M O, Clarkson C, Potsdam, NY

Brierly, Joseph Edward, Mayfair Realty, Livonia, MD

Brooks, Michael Lynn, Rt No 4, Box 59, Greenville, TN

Buxton, Mary M, Ball St U, Muncie, IN

Campbell, Charles J, U of Ark, Fayetteville, AR

Campbell, Robert, $\mathrm{U}$ of Mich, Ann Arbor, MI

Caruso, Jeffrey L, U of Chicago, Chicago, IL

Charalambous, Michael Georgiou, Dept of Math, Zaria, Niger

Cox, Zach D, Charity Jr H S, Rose Hill, NC

Crstici, Borislav, Str 12 Aprolic 1961 NR 6, Timisoara (3), Rumania

Dastrange, Nasser, Pahlavi U, Shiraz, Iran

Davis, Ted S, U of Wash, Seattle, WA

Diaz, Alfonso A, White Sands Missile Range, Las Cruces, NM

Donaldson, John L, Kent St U, Kent, OH

Douglass, Steven Allen, Lawrence U, Appleton, WI

Doyle, Jon, MIT, Cambridge, MA

Elmore, Steven R, Ball St U, Muncie, IN

Evans, Elliott L, U of Tenn, Knoxville, TN

Fitz Patrick, Robert, NJ State Dept of Trans, Trenton, NJ

Fix, G J, Carnegie-Mellon U, Pittsburgh, PA

Fowler, Gary O, 1854 Blovin Ave, Baton Rouge, LA

Fromme, Joseph A, U of Nev, Las Vegas, NV

$\mathrm{Fu}$, Yen-Tzu, Ind St U, Evansville, IN

Funderburk, E James Iii, Northern Ill U, DeKalb, IL

Gagrat, Mani S, Coors Porcelain, El Cajon, CA

Ghulmiyyah, Sari, Marjoyoun, Lebanon

Gomez Martinez, Guillermo Leon, Tech Hochschule, (A-1040) Wien, Austria

Greene, David E, Aerospace Med Res Lab, Wright-Patterson AFB, OH

Habib, Jan Dark, Ghannoum St, Shaar Bldg, Shiyah, Lebanon

Hammond, John A Jr, 3780 N Montana Ave, Helena, MT

Hayward, Thomas J, U of Md, College Park, MD

Hoft, Margret H, U of Mich-Dearborn, Dearborn, MI

Hwang, George R, U of Fla, Gainesville, FL

Im Hof, Hans Christoph, UCB, Berkeley, CA

Ivanovski, Novak, Elektromasinski Fakultet, Skopje (91000), Yugoslavia

Jager, Thomas L, Calvin C, Grand Rapids, MI

Johnstone, P T, U of Chicago, Chicago, IL

Jones, Richard B, 1534 Thomas Ave, Charlotte, NC 
Kent, Michael J, Rutgers U, New Brunswick, NJ Khurana, Surjit Singh, U of Iowa, Iowa City, IA

Khuri, Fawaz D, Murr Bldg, Near Municipality, Furn-El-Chebbak, Beirut, Lebanon

Kiffe, Thomas Robert, Tex A \& M U, Dept of Math, College Station, TX

Kohler, Peter, Justus Liebig U, (6300) Giessen, Fed Rep Germany

Kovacs, Anton Amos, Dept of Math, Santa Barbara, CA

Lambrinos, Panagiotis, Aristotle $U$ of Thessaloniki, Thessaloniki, Greece

Lange, Ridgley, St Andrew's School, Middletown, MD

Lin, Chiu-Tze Lin, U of Ill, Urbana, IL

Loomis, Irene $\mathrm{H}, \mathrm{U}$ of Ala in Birmingham, Birmingham, $\mathrm{AL}$

Marcus, Leo G, UCSB, Santa Barbara, CA

Mcclaran, Virgil R, East Texas Baptist C, Marshall, TX

Melvin, Peter J, U of Ill, Urbana, IL

Mizrahi, Abshalom, 541 Illinois Ave, Glenwood, IL

Mogollon, Ramon E, Inst Univ Pedagogico, Barquisimeto, Venezuela

Mohammadzadeh, Majid, Revolution Corps U, Tehran, Iran

Mortimer, Michael E, Glasgow C of Tech, Glasgow (G4 0BA), Scotland

Mosier, Ronald G, Chrysler, Highland Park, MI

Neumann, Michael, $U$ of Nottingham, Nottingham (NG72RD), England

Nevai, Paul G, U of Wis, Madison, WI

Newton, Mary Elizabeth, St Louis U, St Louis, MO

Norman, Richard G, Columbia Union C, Takoma Park, MD

Ono, Steven G, Natl Inst of Health, Bethesda, MD

Pavlovic, Velimir P, Belgrade U, Belgrade, Yugoslavia

Petricka, Russell J, Carleton C, Northfield, MN

Phoha, Shashi, Tulane U, New Orleans, LA

Poritz, Darwin Holleman, U of Western Ontario, London, Ontario Can

Rabinowitch, Royden, Canal St, P O Box 210, New York, NY

Rajput, Balram S, U of Tenn, Knoxville, TN

Ratner, Marina, UCB, Berkeley, CA

Rault, Jean-Claude L G, Thomson-CSF, (75015) Paris, France

Razzaghi, Mohsen, Pahlavi U, Shiraz, Iran

Regev, Amitai, U of Chicago, Chicago, IL

Riley, Thomas K, Hendrix C, Conway, AR

Risen, Edith E, 455 Mountain View St, Oregon City, OR

Roseman, Dennis, U of Iowa, Iowa City, IA

Rukhin, Andrew L, Opotchinina H.15 A.72, Leningrad (199106), U S S R

Sathaye, Avinash, $\mathrm{U}$ of $\mathrm{Ky}$, Lexington, KY

Schiele, Warren E, Bridgewater-Raritan Reg Sch Dst, Raritan, NJ

Schmidt, Brian K, Notre Dame U, Notre Dame, IN

Sedinger, Harry T, U of Portland, Portland, OR

Setzer, Bennett, U of Ill at Chicago Circle, Chicago, IL

Shelly, John W, Rensselaer Polytech Inst, Troy, NY

Shevchenko, V I, Gomelskii Gos U, Gomel, U S S R

Simms, John C M, Rockefeller U, New York, NY

Strong, Lawrence D, U of S C, Allendale, SC

Sullins, Kenneth W, Croswell-Lexington Commun Schools, Croswell, MI

Surabian, Cynthia G, Simmons C, Boston, MA

Swanberg, Katherine, Internatl Services, Indialantic, FL

Swartout, Leslie E, 4361 Wheeler, Box 120L, Houston, TX

Tabesh, Yahya, Syracuse U, Syracuse, NY

Taylor, Michael E, CUNY, Ctr at Stony Brook, Stony Brook, NY

Telage, Donald Nicholas, U of Del, Newark, DE

Thomas, David, IBM, Owego, NY

Tosques, Mario, Istituto Matematico-Pisa, (56100) Pisa, Italy

Wagon, Stanley, Smith C, Northampton, MA

Wainger, Stephen, Inst for Adv Study, Princeton, NJ

Walker, John Q Ii, 26 Lakewood, Edwardsville, IL

Wells, Larry David, Western Mich U, Kalamazoo, MI

Wilkin, Jo Ann, Miami U, Oxford, OH

Yiu, Yu-Hung Paul, U of Hong Kong, Hong Kong

Zabell, Sandy Lew, U of Chicago, Chicago, IL

Zdravkovska, S, Math Inst, (91000) Skopje, Yugoslavia 
Zigler, William R, BDM, Las Vegas, NV

Zini, Eliahou, Israel Inst of Tech, Haifa (32000), Israel

\section{Membership Under Reciprocity}

Australian Mathematical Society: Brent, Richard P

Dansk Matematisk Forening: Olesen, Dorte Marianne

Gesellschaft fur Angewandte Math und Mechanik: Freud, G

Israel Mathematical Union: Cohn, Harry; Korenblum, Boris; Lichtman, Alexander; Melkman, Awraham Aharon

London Mathematical Society: Cosgrove, John B; Lloyd, Noel G; Robson, J Christopher; Sharma, Chandra Shekhar; Solomon, R C

Polskie Towarzystwo Matematyczne: Baron, Karol

Sociedade Brasileira de Matematica: Pisanelli, Domingos

Societe Mathematique de Belgique: Remans, Andre

Societe Mathematique de France: Kosmann, Yvette

Suomen Matemaattinen Yhdistys: Londen, Stig Olof

Unione Matematica Italiana: De Michele, Leonede

Wiskundig Genootschap: Van Wouwe, J M; Vervaat, Wim

\section{Nominees of Institutional Members}

Acadia Univ: Bustin, V Gary; Chipman, Frederic H; Pulsifer, Jim; Wolfe, Joseph H American Univ: Wettingfeld, Robert W

Andrews Univ: Hill, Kathy L; Razzouk, Anees Jacob

Angelo St Univ: Herridge, Carolyn Ann

Arizona St Univ: Gordon, Michael B; Jones, Alan S; Morris, Jeanne J; Neiers, Michael E;

Ostergren, Steven; White, Robert G; Wyatt, Leslie E; Zimmerman, Martin R

Armstrong St Coll: Findeis, John

Berea Coll: Lohrasbpour, Esfandiar; Tamayo, Gwen J

Boise St Univ: Dodson, Kathleen A

Bowling Green St Univ: Bardwell, Maureen A; Kellermeier, John H; Putt, Harold L

Brooklyn Coll, CUNY: Bergen, Jeffrey M; Calcaterra, Robert; Keller, Arthur M

Brown Univ: Hwang-Ma, Shu-Yin; Kotzen, Robert

Calif St Univ, Northridge: Abram, Thomas Joseph; Clemens, Shawna; Litzenberger,

Richard A; Rackusin, Jeff

Carnegie-Mellon Univ: Cha, Anna En-Tzu; Detig, Diane Marie; Farinacci, Martha Louise;

Greer, Kenneth L; Grefenstette, John J; Littler, John C; Neta, Beny; Osgood, Brad G;

Stanley, Keith R; Yatomi, Chikayoshi

Catholic Univ of America: Bernal, Javier

City Coll, CUNY: Gartner, Susan; Leon/Ring, Rochelle H; Leung, Peter;Peikes, Meyer J; Warshajchik, Jack

Clarion St Coll: Caiazza, Mary Ann; Ossesia, Michel G

Clark Univ: Ledbetter, Carl

Colorado St Univ: Longsine, Dennis Earl; Quesenberry, David W; Willis, J Michael

Concordia Coll, Moorhead: Helsa, Todd; Heuer, Karl W

Cornell Univ: Bell, John Bruce; Blank, Brian Evan; Brady, Stephen; Gatsonis, Constantine

A; Goldstein, Norman J; Hsiao, Cheng-Tan; Immerman, Neil; Kazez, William H;

Nelson, Robert K; Pearlman, Michael D; Pimm, David John; Sammon, Peter H; Sheu,

Wen-Lian; Trigg, Randall $\mathrm{H}$

Dartmouth Coll: Burkhart, Richard H; Gordon, Jean L

Drexel Univ: Doyle, Daniel A; Soyka, Michael G

Duke Univ: Blake, Lewis D III; Clote, Peter G; Holder, Ernest Jefferson; Israel,

Maximiano M Jr; Mason, Ronald Earl; Nelson, James D; Norman, Paul D; Penkava,

Michael R; Smith, Milton Dale; Tiballi, Terry; Tso, Chak Yuen; Williams, Dorothy M

Duquesne Univ: Che, Fang; Kabana, Alfred C; Mccann, Matthew F; Wills, Charles A

East Carolina Univ: Joyner, Robert N; Stocks, Debra L

East Texas St Univ: Hicks, Jerry L

Eastern Montana Coll: Berg, William Albert; Mckinley, William S

Eastern New Mexico Univ: Holt, Cindy C; Large, Marilyn L; Truitt, David H; Wood, Jimmy

Eckerd Coll: Connor, Jeffrey S; Dennis, Michael S; Farrer, Brian H; Halchin, Judy D

Fairleigh Dickinson U, Teaneck: Chrysostomou, Andreas K 
Franklin \& Marshall Coll: Hershorin, Gary M; Lee, Robert H; Smith, Jack S

Ft Hays Kansas St Coll: Riekenberg, John M

George Mason Univ: Hall, Barry C; Hutchins, Nan; Preiss, Kirk Alan

Georgetown Univ: Centola, Maryjane $\mathrm{J}$

Georgia Inst of Tech: Hubbard, Elaine M; Johnson, Charles M Jr; Siegrist, Kyle T

Harvard U: Angelo, Paul E; Dilek, Rita; Gabber, Ofer; Ghent, Robert C; Gillet, Henri; Jochnowitz, Naomi G; Lang, William E; Olver, Peter J; Stevens, Glenn Howard; Ticciati, Caroline $M$

Hendrix Coll: Berry, Leslie Lynne

Hobart \& William Smith Coll: Albright, Richard A; Smolowitz, Lawrence H

Hope Coll: Donnelly, Stephen J

Humboldt St $U$ : Jackson, Lynn; Mousouvis, Nick

Hunter Coll, CUNY: Sacolick, Sylvan Louis

Idaho St Univ: Bush, William Joseph Jr; Carlson, Kenneth E Jr; Hymas, James Andy

Indiana Univ at Bloomington: Latia, Andre

Indiana Univ of Pennsylvania: McBridge, Ronald L

Indiana Univ-Purdue Univ: Chong, Susan; Huffman, G David; Hutton, Lucreda A; Luke, Jon C

Inst for Advanced Study: Burns, Daniel M Jr; Cwikel, Michael; Deodhar, Vinay Vithal; Enright, Thomas J; Freedman, Michael H; Harrison, Jenny; Hausmann, Jean-Claude; Kimura, Tatsuo; Nori, Madhav V; Weisfeiler, Boris

Iowa St Univ: Chang, Yen Fook; Gray, Timothy A; Jones, Kathryn A; Pothoven, Keith E Johns Hopkins U: Delcher, Arthur Leland; Meuser, Diane; Shieh, Shyan-Chern

Juniata Coll: Cauffman, John M

Kent St U, Kent: Beggs, Robert D; Benander, Barbara Ann; Creekmore, Joseph W; Giel, Charles T; Ruttan, Arden G

Lakehead Univ: Ahmad, Afaq; Cantor, Rita M; Chew, Kok-Thai; Ranta, Martin J

Lamar Univ: Mades, John W

Lehigh Univ: Anderson, David B; Bacon, Edward F; Bingham, Ann D; Klawitter, Warren A; Schiefer, Randall C; Tanzini, Joseph P; Vivian, Judith M; Wilder, Robert G

Louisiana Tech Univ: Mcmath, John S

Macalester Coll: Clipson, Michael A; Johnson, Daniel P

Mankato St Coll: Geurs, Robert B

Marquette Univ: Brooks, Marie A; Eibl, Marie E; Liang, Sysh-Cherng

Marshall Univ: Harrison, Donna S; Pollock, David Wayne; Thomas, Joseph H

Massachusetts Inst of Tech: Brodsky, David A; Christiansen, Edmund; Diamond, Harvey; Forsythe, Keith W; Gutmann, Samuel Chaim; Kay, Joseph A; Krevitt, James S;

Morin-Strom, Karl A; Pannatoni, Ronald F; Rojas-Viggiani, Lucas V; Sultan, Fareena

McGill Univ: Mackenzie, Kenneth W

Memorial $U$ of Newfoundland: Acreman, Dennis; Meade, Barbara A

Memphis St Univ: Coke, Larry Randall; Jea, Hae-Pyng; Parrott, Mary E; Siler, William L

Michigan St Univ: Assiff, Thomas C; Chin, Jenny Chui-Ping; Daepp, Ulrich; Danon,

Victor; Deneen, Linda L; Douglass, Beth A; El-Mandouh, Mohamed S; Jurzysta, Sonja

H; Kusnerus, Wendell G; Lindenfeld, Israel; Ma, Frank C; Naik-Nimbalkar, Uttara V;

Novak, Schu R; Rogers, Julie Ann; Shaughnessy, J Michael; Witter, Lester J

Middlebury Coll: Bielli, Ronald R

Minot St Coll: Babb, James H

Mississippi St Univ: Connors, Jan; King, Herbert A; Smith, Wanda W

Montana St Univ: Hamllik, Thomas B; Johnston, Bruce W; Weinstein, Larry Hale

Moorhead St Univ: Carlson, Brian Lowell; Schott, Steven Allen

Mt Allison Univ: Prince, W Steven; Rach, Marion E

New Jersey Inst of Tech: Dios, Rose; Keith, Michael

New York Univ, Courant Inst: Burman, David Y

North Carolina St Univ: Feathers, Gordon R; Kim, Sun-Hae; Lehmann, Joel P; Tzung, Fu-Chien

North Texas St Univ: Dorsett, Charles; Miller, Robert P; Voas, Charles H

Northeastern Illinois Univ: Patricelli, Anthony A; Sheridan, Ardis L

Northern Michigan Univ: Constance, B A; Kulisheck, Meredith A

Oakland Univ: Blumberg, Carol Joyce; Peterson, Edward Lawrence

Penn St Univ, University Park: Bond, Charles S; Hager, James Andrew; Halpin, Patrick; Jackson, Madeline; Lang, Sheau-Dong; Lefkowitz, Jerry M; Obaid, Evelyn E; Pacitti, Patricia Anne; Pineault, Wayne B; Whittington, Keith E

Polytechnic Inst of New York: Freedman, Roy Stuart; Kamienny, Sheldon; Stucker, Aaron I 
Princeton Univ: Assadi, Amir Hossein; Chess, Daniel S; Cybenko, George; Dang, Dinh Lanh; Dummit, David; Jacob, William Burkley; Kerckhoff, Steven P; Kohn, Robert V; Ling, Wensor; Mahler, Howard C; Myers, Piers; Shapiro, Helene; Shucker, David S; Steiner, Michael

Purdue Univ: Bradley, Patrick J; Gupta, Omprakash K; Roberts, Gary B; Woolford, Samuel W

Purdue Univ, Calumet Campus: Maicher, Joan M; Marecek, Lynn Marie; Thiel, T J

Rensselaer Polytech Inst: Babchyck, Barry Myer; Barry, Marian R; Hegner, Nancy S; Pate, Edward F; Watson, John Gordon; Yumba, Nkasa E

Rice Univ: Stewart, Mark D

Rutgers Univ, New Brunswick: Baxter, Nancy Hood; Felcon, Francis Xavier; Gillam, William Robert; Hoyle, Stephen; Hutson, Holmes Leroy Jr; Kurtz, Douglas S; Robbin, Ira; Schwartz, Charles Freuld; Siege, Alan; Silva, Maria Welleda; Silverstein, Anna R; Stafford, Richard M; Tutinas, Bernadette Romualda; Weiss, Michael; Wilson, Ted C

San Diego St Univ: Heller, Steve; Hinckley, Diane Rita; Hopper, Tom; Rittenhouse, Robert G

Seton Hall Univ: Rosell, Bard Maurice

So Dakota Sch of Mines \& Tech: Loken, Marc C; Martin, John Farrell

South Dakota St Univ: Monahan, Maurice Lee

Southeastern Mass Univ: Medeiros, Carol A

Southern Ill U, Edwardsville: Hashimi, Jamil R; Kistner, James E; Vassier, Stephen B; Veile, Darrell R

Southern Methodist Univ: Daniel, Patricia Kay; Davis, Cassandra C; Haughey, James C; Mitchell, Napoleon; Moore, John P; Roberts, Marilynn Lee

Southwest Missouri St Univ: Baker, Jimmy Dole; Fishburn, Diane J

St Ambrose Coll: Chung, Sei-Jong

St Francis Coll: Kudrick, John M

St John's Univ: Schiffman, Jay Lawrence; Woods, Patrick B

St Mary's Coll of Maryland: Walker, Harry D

Stanford $U$ : Cameron, Rory H; Chen, Jin-Tzu; Franzblau, Alfred; Griffin, Edmond E II; Hergesell, Gerhard A; Kimura, Yumi; Koh, Kyewon; Levin, Stewart A; Marks, Daniel G; Rea, Marilyn E; Robson, Robert O; Sallustro, Bruno; Sexton, Harlan B; Smith, Marian; Stillman, Stephen Michael

Stephen F Austin St Univ: Fuller, Darlene S; Scott, Barbara Ann; Shepard, Jane Staats; Walding, Judy Kay

Stetson Univ: Gaylord, John M

Stevens Inst of Tech: Mehnert, Elizabeth F

SUNY, Coll at Brockport: Bobon, Vincent T; Decicca, Joseph W; Himelein, James A Jr; Manogue, Susan $\mathbf{M}$

SUNY, Coll at Oswego: Chrissohoos, Nikos P; Gill, Thomas M; Graci, Craig A

SUNY, Ctr at Stony Brook: Dodson, B

Susquehanna Univ: Tyler, Robert L

Syracuse Univ: Blair, Howard A; Burt, Earl C; Cairoli, Louis H; Mcevoy, Jeanne E; Melo, J Ulisses; Palumbo, Thomas M; Urner, Robin Lee; Weiss, Harold M

Temple Univ: Atacik, Sermin; Brubaker, Jere C; Heller, Esther A; Mccleary, John H; Smith, Robert C Jr; Snyder, James M

Tennessee Tech Univ: Randolph, Lisa Lynn

Texas Christian Univ: Morgan, Ronald Lewis; Reagor, Mary Pensworth

Tufts Univ: Hajj, Jack P

Tulane Univ: Beatrous, Frank H; Brown, Peter N Jr; Buttner, Wolfram R; Frame, Michael Lee; Frias, Simon; Haaland, Perry Dean; Messa, Kenneth C; Orr, Dalton E; Ouyoung, Kuong Lin; Pick, Edward C; Przygocki, Tony; Spangler, T C Jr; Spurr, Michael J

$U$ of Colorado, Boulder: Barraza, Daniel M; Bateson, Carol Ann; Cervantes, Rafael; Everts, Franklin F; Lawson, Reginald D; Pedersen, Paul S; Rassbach, Walter B; Reid, Walter $\mathbf{M}$

$U$ of Ill at Chicago Circle: Ash, Arlene S; Foody, Walter M; Hughes, Michael J; Leonetti, Richard A; Weibull, Thomas

U of Ill at Urbana-Champaign: Alter, Judy F; Bingham, Nicholas H; Bitner, James R; Carlson, Robert P; Currier, Robert J; Gajendragadkar, Dilip S; Gunter, Berton H; Hopkins, Mark R; Pinzur, Laurence A; Redmond, David Brendan; Savage, Carla Diane; Thomas, Mark A; Timoney, Richard M; Turgi, Mary

$U$ of New Brunswick, Fredericton: Boyer, Steven Patrick; Chen, Tzer-Lin 
$U$ of Wisc, Madison: Wolf, Thomas R

$U$ of Wisc, Milwaukee: Gerasch, Thomas E; Mikkelson, Ruth L; Ristow, Allan W

$U$ of Wisc, Parkside: Funk, Debra $\mathrm{J}$

Univ of Alabama-Birmingham: Ubben, Rebecca L

Univ of Alabama-Tuscaloosa: Copeland, Charles Henry; Vonbachhaus, Anton L W; Williams, Sarah

Univ of Arizona: Anderson, Ian M; Baumeister, Richard; Chumpitaz, Mauro; Goodwin, William J; Johnson, William A; Khan, A F M Khodadad; Thompson, Donald Mark Univ of British Columbia: Gentle, Ronald S; Mcleod, Kenneth I; Taylor, Gordon E

Univ of Calgary: Mbuntum, Francis Fai; Nowakowski, Richard J; Toporowski, Stanley F

Univ of Calif, Davis: Barcellos, Anthony; Cheng, Pi-Yun; Hijab, Omar B; Jasiulek, Joachim; Kaiser, Lee David; Melcon, David Parnak; Rusnak, Kurt

Univ of Calif, Los Angeles: Bell, Jonathan G; Brietzke, Eduardo; Clapp, Edward;

Cunningham, James; Davenport, C Brent; Fagarasan, John Theodor; Fitzgerald, Robert

W; Freed, Bruce E; Freedman, Jeffrey S; Freiling, Chris; Gilstein, C Zacharay; Hughes,

Kimball; Juo, Lynn; Kosaki, Hideki; Levin, Alan Charles; Malkus, Katherine A;

Patrusky, Julie M; Rustin, Garth H; Schatz, Mary Christine; Spagnuolo, John Nicholas; Stevens, Doug L; Wong, Oy-Yee

Univ of Calif, Riverside: Bain, Michael L; Conyers, Steven James

Univ of Cincinnati: Dobur, Olsa; Hollingsworth, Mark B; Lyzzaik, Abdallah Khalil

Univ of Conn, Storrs: Chiang, Tsyh-Yun; Riley, John H; Su, Sung-Woo

Univ of Dayton: Bachmann, Michael R; Koepplinger, George Kenneth

Univ of Delaware: Alexiades, Vasilios

Univ of Detroit: Bukowski, Mary O; Meyer, Victor Arthur; Zukowski, C C

Univ of Florida: Atkins, Robert H Jr; Howorka, Edward R; Yamamoto, Yutaka

Univ of Georgia: Chen, Tsann-Hoa; Kuhn, Stephen Walter; Lynch, Thomas Emile; McCabe,

Pamela Ann; Patwardhan, Mangal; Younis, Rahman Mahmoud

Univ of Hartford: Mingrone, Joseph R; Russell, Lee A

Univ of Idaho: Cambareri, Salvatore; Sell, David E

Univ of Lethbridge: Morrell, Lynne B

Univ of Maryland, College Park: Creegan, Paul Francis Jr; Graubard, Barry Ira; Majer, Vaclav

Univ of Miami: Bartick, Philip R

Univ of Michigan, Ann Arbor: Brown, Johnny E; Clark, Curtis; Exoo, Geoffrey; Hagwood,

Robert C; Han, Chong-Kyu; Hawkins, William Anthony Jr; Hsu, Derbiau F; Oh, Hae

Soo; Rees, Howard David; Robinson, Michael L; Smith, Randolph G; Squires, William

A; Tsao, Anna; Volkman, Thomas Rudolph

Univ of Minnesota-Duluth: Howe, Paul L; Marttila, Charles A

Univ of Mississippi: Allen, Lynda L; Caldwell, Doris Daonne; Chin, Jerry M; McClanahan, Ray A

Univ of Missouri, Kansas City: Farrell, Frances K

Univ of Missouri, St Louis: Monachella, Mary Beth; Vinner, Hava

Univ of Montana: Conner, Max L

Univ of $N C$ at Greensboro: Mills, Douglas L; Wagoner, Bobbie D

Univ of Nebraska at Lincoln: Carroll, John; Fischer, Thomas S; Midgarden, Bette Gene

Univ of New Mexico: Sanchez, E Andrew; Trucano, Timothy Guy

Univ of Notre Dame: Barry, Michael James; Bouma, Herman J; Callan, C David;

Harkleroad, Leon Wade; Miller, Timothy James; Weyland, Nicholas John; Wong,

Pit-Mann

Univ of Ottawa: Awai, Andrew Equan; Desale, Govindrao Bhikanrao; Herrera, Ricardo; Li, Yiu-Kwan K; Tapia, Claudio E; Violette, Donald

Univ of Pennsylvania: Cole, Paul Dana; Debaun, David R; Evans, Bruce D; Gass, Michael

D; Gnanamgari, Sakunthala; Hager, Mark; Price, Geoffrey L; Schmidt, Frank W;

Simpson, Janet; Singh, Om Prakash; Solomon, Daniel L; Winter, Sara B

Univ of Regina: Marley, John A; Robinson, Gordon C

Univ of Rhoue Island: Levasseur, Kenneth M; Murthy, Narayan S

Univ of Rochester: Varilly, Joseph C

Univ of Santa Clara: Coletti, Neil B; McCurley, Kevin Snow

Univ of Saskatchewan: Basti, Mehran; Hamoud, G A; Hegde, Shankar T

Univ of South Carolina: Daffer, Peter Zito; Lee, Arthur C H; Matthews, Manton McCutchen

Univ of Southern Calif: Franks, Don G; Gleicher, Ben; Jameson, James R; Rosales, Silvia Huerta; Strand, Peter G 
Univ of Tennessee, Chattanooga: Beard, Amy Joanne; Harrison, Charles Lester

Univ of Texas at Arlington: Bronson, Evin Joyce; Hallmark, James C; Radhakrishna, Atur $\mathrm{V}$

Univ of Texas at Austin: Best, Ralph L Jr; Cohen, Martin P; Hayes, Linda J; Lindsay, James W; Rooney, Martin M; Shaw, Terry D; Wolfe, Michael David

Univ of Texas at El Paso: Rojo, Javier J; Russell, Carol S

Univ of Toronto: Brunstad, Solmund G; Chalmers, Bruce A; D'Angelo, Mario P R; Davies, Peter C; Gomez, Francisco; Mingarelli, Angelo Bernardo; Monson, Barry R; Segall, Simon A; Wong, Man Wah

Univ of Tulsa: Aguilar, Jesse; Bennion, Patricia Ann; Ogle, Robert A; Williams, James L Univ of Utah: Hoover, John Craig; Kearfott, R Baker; Li, Hilda Chu; Lund, John R; Matsumoto, Allen S; Tucker, Carolyn Connell

Univ of Virginia: Foiles, William Clifton; Reid, David Hart; Schneider, Michael H

Univ of Waterloo: Cheng, Kuang-Fu; Ebanks, Bruce R; McCarthy, David; Scott, Philip J; So, Ying C; Szafron, Duane A

Univ of Wyoming: Gaeth, Gary John; Harrington, John R; Miel, George

Valdosta St Coll: Outlaw, Charlotte J; Roberts, William G

Vanderbilt Univ: Collier, Manning Gary; Edwards, Jo A; Fuqua, Mark A; Sanders, Robert Louis; Smith, Patricia Anne; Wilson, Charles Richard

Vassar Coll: Cleveland, Linore $\mathrm{H}$

Villanova Univ: Fini, Robert L; McHugh, Joseph Francis; Meketon, Marc Stuart

Virginia Commonwealth $U$ : Brasington, Diane F; Davis, Cheryl M; Fulton, M L; Henley, Oley Adolphus

Washington \& Lee Univ: Rigby, David $\mathrm{P}$

Washington Univ: Hemler, Michael Lee; Kline, Robert M; Province, Michael Arthur

West Georgia Coll: Bagwell, George Dilmus; Bogue, Harriet H

Western Illinois Univ: Faraca, Daniel Louis; Khan, Nasir H; Prosser, Mark F; Stuff, Mark A

Wheaton Coll: Guess, Frank M; Sweeney, Sally Ann

Wichita St Univ: Besser, John D; Bouska, Charles A; Noller, Gary L

Wilkes Coll: Mantione, Ross L; Weisman, David B Jr

Worcester Polytechnic Inst: Gurlitz, Thomas Richard; Willits, Roy C

Wright St Univ: Aldrich, Kenneth A; Glass, Betty C; Lee, Dailing

York Univ: Burbulla, Dietrich; Kasimos, Nikolas; Li, W K; Philip, George

Youngstown St Univ: Bilas, Barbara Ann; Doles, Gary L 